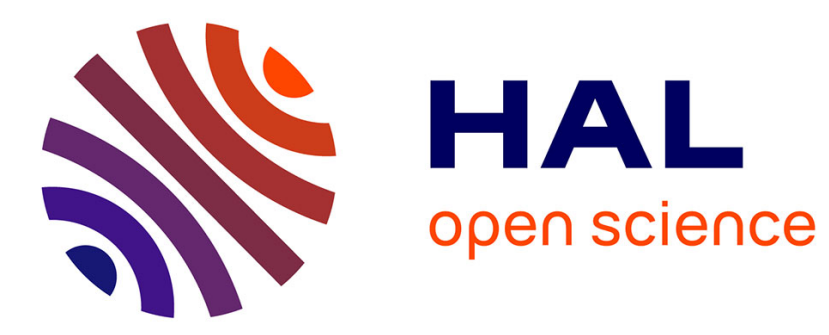

\title{
Some linear fractional stochastic equations
}

Ivan Nourdin, Ciprian A. Tudor

\section{To cite this version:}

Ivan Nourdin, Ciprian A. Tudor. Some linear fractional stochastic equations. Stochastics: An International Journal of Probability and Stochastic Processes, 2006, 78 (2), pp.51-65. 10.1080/17442500600688997 . hal-00013903v2

\section{HAL Id: hal-00013903 https://hal.science/hal-00013903v2}

Submitted on 10 Mar 2006

HAL is a multi-disciplinary open access archive for the deposit and dissemination of scientific research documents, whether they are published or not. The documents may come from teaching and research institutions in France or abroad, or from public or private research centers.
L'archive ouverte pluridisciplinaire HAL, est destinée au dépôt et à la diffusion de documents scientifiques de niveau recherche, publiés ou non, émanant des établissements d'enseignement et de recherche français ou étrangers, des laboratoires publics ou privés. 


\title{
Some linear fractional stochastic equations
}

\author{
Ivan Nourdin* \\ LPMA, Université Pierre et Marie Curie Paris 6, \\ Boite courrier 188, 4 Place Jussieu, 75252 Paris Cedex 5, France \\ nourdin@ccr.jussieu.fr \\ Ciprian A. Tudor \\ SAMOS/MATISSE, Centre d'Economie de La Sorbonne, \\ Université de Panthéon-Sorbonne Paris 1 \\ 90, rue de Tolbiac, 75634 Paris Cédex 13, France \\ tudor@univ-paris1.fr
}

\begin{abstract}
Using the multiple stochastic integrals we prove an existence and uniqueness result for a linear stochastic equation driven by the fractional Brownian motion with any Hurst parameter. We study both the one parameter and two parameter cases. When the drift is zero, we show that in the one-parameter case the solution in an exponential, thus positive, function while in the two-parameter settings the solution is negative on a non-negligible set.
\end{abstract}

Key words: Fractional Brownian motion, fractional Brownian sheet, multiple stochastic integral, Girsanov transform.

2000 Mathematics Subject Classification: 60H05,60G15,60G18.

\section{Introduction}

The significant amount of applications where the fractional Brownian motion (fBm) is used led to the intensive development of the stochastic calculus with respect to this process and its planar version. The study of stochastic differential equations (SDEs) driven by a

\footnotetext{
${ }^{*}$ Corresponding author
} 
fractional Brownian motion followed in a natural way. Let us consider $\left(B_{t}^{\alpha}\right)_{t \in[0, T]}$ a fBm with Hurst parameter $\alpha \in(0,1)$. Essentially, one can consider the SDE

$$
d X_{t}=\sigma\left(t, X_{t}\right) d B_{t}^{\alpha}+b\left(t, X_{t}\right) d t
$$

in two ways:

- the pathwise (Stratonovich) type (that is, the stochastic integral is considered in a pathwise sense);

- the divergence (Skorohod) type (that is, the stochastic integral is of divergence type).

The first type of equations, which includes the rough paths theory and the stochastic calculus via regularization, can in general be solved by now standard methods. We refer, among others, to [1, 4, 6, 8, 12, 13, 16, 21]. The second type (Skorohod stochastic equations) is more difficult to be solved. Even in the standard Brownian motion case (corresponding to $\alpha=1 / 2$ ), we have an existence and uniqueness result only in two situations:

- when $\sigma\left(s, X_{s}\right)=\sigma(s) X_{s}$ with $\sigma(s)$ random: we then use an anticipating Girsanov transform, see [2],

- when $\sigma\left(s, X_{s}\right)=\sigma(s) X_{s}$ and $b\left(s, X_{s}\right)=b(s) X_{s}$ with $\sigma, b$ two deterministic functions: we can then use a method based on the Wiener-Itô chaotic expansion.

This second approach will be considered in our paper. We will consider the stochastic equation

$$
X_{t}=1+\int_{[0, t]} a X_{s} \delta B_{s}^{\alpha}+\int_{[0, t]} b X_{s} d s
$$

where $a, b$ are real numbers and the stochastic integral is understood in the Skorohod sense. We first prove existence and uniqueness results in the one-parameter case (that is when $t \in[0, T]$ ) and in the two-parameter case (that is when $t \in[0, T]^{2}$ and with $B^{\alpha}$ replaced by a fractional Brownian sheet $W^{\alpha, \beta}$ with Hurst parameters $\left.\alpha, \beta\right)$.

Of course, the fact that the above linear equation can be solved by using Wiener-Itô multiple integrals is not very surprising; it has already used in [17] for $\alpha>\frac{1}{2}$. Nevertheless, we have to check some new technical aspects like: the proof of the case $\alpha \in\left(0, \frac{1}{2}\right)$ or the proof of the two-parameter case for any Hurst parameters $\alpha$ and $\beta$.

More surprising is, as in the case of the standard Brownian sheet (see [15]), the behavior of the solution of (2) when the drift $b$ is zero: in the one-parameter case, the solution is an exponential, hence positive, function while in the two-parameter case the solution is negative on a non-negligible set. We also mention that, comparing to the standard case when the Hurst parameters are $\frac{1}{2}$, new techniques like fractional Girsanov theorem and estimations of fractional norms of the kernels appearing in the chaotic expression of the solution of (21), are here needed. We refer to [19] for applications of stochastic equations driven by fractional Brownian sheet to statistics. 
We organized our paper as follows. Section 2 contains some preliminaries on fractional Brownian motion and fractional Brownian sheet. In Section 3 we study the existence, the uniqueness and the properties of the solution of equation (2) in both one-parameter and two-parameter cases. Section 4 contains a technical proof.

\section{Preliminaries}

Consider $\left(B_{t}^{\alpha}\right)_{t \in[0, T]}$ a fractional Brownian motion (fBm) with Hurst parameter $\alpha \in(0,1)$ and let us denote by $R^{\alpha}$ its covariance function

$$
R^{\alpha}(s, u)=\frac{1}{2}\left(s^{2 \alpha}+u^{2 \alpha}-|s-u|^{2 \alpha}\right)
$$

for every $s, u \in[0, T]$. It is well-known that $B^{\alpha}$ admits the Wiener integral representation

$$
B_{t}^{\alpha}=\int_{0}^{t} K^{\alpha}(t, s) d W_{s}
$$

where $W$ denotes a standard Wiener process and

$$
K^{\alpha}(t, s)=d_{\alpha}(t-s)^{\alpha-\frac{1}{2}}+s^{\alpha-\frac{1}{2}} F_{1}\left(\frac{t}{s}\right)
$$

$d_{\alpha}$ being a constant and $F_{1}(z)=d_{\alpha}\left(\frac{1}{2}-\alpha\right) \int_{0}^{z-1} \theta^{\alpha-3 / 2}\left(1-(\theta+1)^{\alpha-1 / 2}\right) d \theta$.

By $\mathcal{H}(\alpha)$ we will denote the Hilbert space associated to $B^{\alpha}$ defined as the closure of the linear space generated by the indicator functions $\left\{1_{[0, t]}, t \in[0, T]\right\}$ with respect to the scalar product

$$
\left\langle 1_{[0, t]}, 1_{[0, u]}\right\rangle_{\mathcal{H}(\alpha)}=R^{\alpha}(t, u) .
$$

The structure of $\mathcal{H}(\alpha)$ depends on the values of the Hurst parameter $\alpha$. Let us recall the following facts:

- if $\alpha \in\left(\frac{1}{2}, 1\right)$, then it follows from [18] that the elements of $\mathcal{H}(\alpha)$ may be not functions but distributions of negative order. Thus it is more convenient to work with subspaces of $\mathcal{H}(\alpha)$ that are sets of functions. A such space is the set $|\mathcal{H}(\alpha)|$ of measurable functions on $[0, T]$ such that

$$
\int_{0}^{T} \int_{0}^{T}|f(u)||f(v)||u-v|^{2 \alpha-2} d u d v<\infty
$$

endowed with the scalar product

$$
\langle f, g\rangle_{|\mathcal{H}(\alpha)|}=\alpha(2 \alpha-1) \int_{0}^{T} \int_{0}^{T} f(u) g(v)|u-v|^{2 \alpha-2} d u d v .
$$

We have actually the inclusions

$$
L^{2}([0, T]) \subset L^{\frac{1}{\alpha}}([0, T]) \subset|\mathcal{H}(\alpha)| \subset \mathcal{H}(\alpha) .
$$


- if $\alpha \in\left(0, \frac{1}{2}\right)$ then the Hilbert space $\mathcal{H}(\alpha)$ is a space of functions contained in $L^{2}([0, T])$. It contains the space of Hölder functions of order $\alpha-\varepsilon$ with $\varepsilon>0$ and it can be characterized by

$$
\mathcal{H}(\alpha)=\left(K^{*}\right)^{-1}\left(L^{2}([0, T])\right)
$$

where the operator $K^{*}$ is given by

$$
\left(K^{*} \varphi\right)(s)=K^{\alpha}(T, s) \varphi(s)+\int_{s}^{T}(\varphi(r)-\varphi(s)) \frac{\partial K^{\alpha}}{\partial r}(r, s) d r .
$$

A fBm being a Gaussian process, it is possible to construct multiple Wiener-Itô stochastic integrals with respect to it. We refer to [14] for general settings or to [17] for the adaptation to the fractional Brownian motion case. We only recall that the multiple integral of order $n$ (denoted by $I_{n}$ ) is an isometry from $\mathcal{U}^{\otimes n}$ to $L^{2}(\Omega)$ where $\mathcal{U}$ is the Hilbert space $|\mathcal{H}(\alpha)|$ if $\alpha \in\left(\frac{1}{2}, 1\right)$ and the Hilbert space $\mathcal{H}(\alpha)$ if $\alpha \in\left(0, \frac{1}{2}\right)$.

We need to introduce the space $D^{c h}$ of stochastic processes that can be expressed in terms of multiple stochastic integrals. That is, we denote by $D^{c h}$ the set of processes $u \in L^{2}(\Omega ; \mathcal{U})$ such that for every $t \in[0, T]$,

$$
u_{t}=\sum_{n \geq 0} I_{n}\left(f_{n}(\cdot, t)\right)
$$

where $f_{n} \in \mathcal{U}^{\otimes n+1}$ is symmetric in the first $n$ variables and

$$
\sum_{n \geq 1}(n+1) !\left\|f_{n}\right\|_{\mathcal{U}^{\otimes n+1}}^{2}<\infty
$$

It follows from [17] (for $\alpha>\frac{1}{2}$ ) or [3], [10] (for $\alpha<\frac{1}{2}$ ) that if $u \in D^{c h}$ then $u$ is Skorohod integrable with respect to the $\mathrm{fBm} B^{\alpha}$ and in this case its Skorohod integral is

$$
\delta(u)=\sum_{n \geq 0} I_{n+1}\left(\tilde{f}_{n}\right)
$$

where $\tilde{f}_{n}$ means the symmetrization of $f_{n}$ with respect to $n+1$ variables. Actually, in the case $\alpha<\frac{1}{2}$ the expression (11) corresponds to the divergence integral in the extended sense (see [3]).

Let us consider now the two-parameter case. Here, $W^{\alpha, \beta}$ is a fractional Brownian sheet with Hurst parameters $\alpha, \beta \in(0,1)$. Recall that $W^{\alpha, \beta}$ is defined as a centered Gaussian process starting from 0 with the covariance function

$$
\begin{aligned}
E\left(W_{s, t}^{\alpha, \beta} W_{u, v}^{\alpha, \beta}\right) & =R^{\alpha, \beta}(s, t, u, v) \\
& :=\frac{1}{2}\left(s^{2 \alpha}+u^{2 \alpha}-|s-u|^{2 \alpha}\right) \frac{1}{2}\left(t^{2 \beta}+v^{2 \beta}-|t-v|^{2 \beta}\right)
\end{aligned}
$$


and it can be represented as

$$
W_{s, t}^{\alpha, \beta}=\int_{0}^{t} \int_{0}^{s} K^{\alpha}(t, u) K^{\beta}(s, v) d W_{u, v}
$$

where $\left(W_{u, v}\right)_{u, v \in[0, T]}$ is a standard Brownian sheet and $K^{\alpha}$ is given by (邨. Denote by

$$
K^{\alpha, \beta}(t, s)=K^{\alpha}(t, u) K^{\beta}(s, v)
$$

and let $\mathcal{H}^{(2)}(\alpha, \beta):=\mathcal{H}^{(2)}$ be the canonical Hilbert space of the fractional Brownian sheet $W^{\alpha, \beta}$. That is, $\mathcal{H}^{(2)}$ is defined as the closure of the set of indicator functions $\left\{1_{[0, t] \times[0, s]}, t, s \in\right.$ $[0, T]\}$ with respect to the scalar product

$$
\left\langle 1_{[0, t] \times[0, s]}, 1_{[0, u] \times[0, v]}\right\rangle_{\mathcal{H}^{(2)}}=R^{\alpha, \beta}(s, t, u, v)
$$

for every $t, s, u, v \in[0, T]$.

By the above considerations, we will have:

- if $\alpha, \beta \in\left(\frac{1}{2}, 1\right)$, the elements of $\mathcal{H}^{(2)}$ may be not functions but distributions. Thus it is more convenient to work with subspaces of $\mathcal{H}^{(2)}$ that are sets of functions. We have actually the inclusions

$$
L^{2}\left([0, T]^{2}\right) \subset|\mathcal{H}|^{(2)} \subset \mathcal{H}^{(2)}
$$

where

$$
|\mathcal{H}|^{(2)}=|\mathcal{H}(\alpha)| \otimes|\mathcal{H}(\beta)|
$$

and $|\mathcal{H}(\alpha)|$ is defined by (的).

- if $\alpha, \beta \in\left(0, \frac{1}{2}\right)$ then the canonical space $\mathcal{H}^{(2)}$ is a space of functions that can be written as

$$
\mathcal{H}^{(2)}=\left(K^{*, 2}\right)^{-1}\left(L^{2}\left([0, T]^{2}\right)\right) \subset L^{2}\left([0, T]^{2}\right)
$$

where $K^{*, 2}$ is the product operator $K^{*} \otimes K^{*}$ and $K^{*}$ is given by (9).

- if $\alpha \in\left(\frac{1}{2}, 1\right)$ and $\beta \in\left(0, \frac{1}{2}\right)$, then $|\mathcal{H}|^{(2)}$ is not a space of functions and we will work with the subspace $|\mathcal{H}(\alpha)| \otimes \mathcal{H}(\beta)$.

Let us denote by $\mathcal{V}$ the Hilbert space: $|\mathcal{H}|^{(2)}$ if $\alpha, \beta \in\left(\frac{1}{2}, 1\right) ; \mathcal{H}^{(2)}$ if $\alpha, \beta \in\left(0, \frac{1}{2}\right)$ and $|\mathcal{H}(\alpha)| \otimes \mathcal{H}(\beta)$ if $\alpha \in\left(\frac{1}{2}, 1\right)$ and $\beta \in\left(0, \frac{1}{2}\right)$.

We can of course consider multiple stochastic integrals with respect to the Gaussian process $W^{\alpha, \beta}$. Here the multiple integral of order $n$, still denoted by $I_{n}$, will be a isometry from $\mathcal{V}^{\otimes n}$ to $L^{2}(\Omega)$. 


\section{Linear stochastic equations with fractional Brownian mo- tion and fractional Brownian sheet}

Let us consider the following stochastic integral equation

$$
X_{t}=1+\int_{0}^{t} a X_{s} \delta B_{s}^{\alpha}+\int_{0}^{t} b X_{s} d s, \quad t \in[0, T]
$$

where $a, b \in \mathbb{R}$ and the stochastic integral above is considered in the Skorohod sense. We will first prove the existence and the uniqueness of the solution of (15), in the space $D^{c h}$. For $\alpha>\frac{1}{2}$ this has been proved in 17].

Proposition 1 The equation (15) admits an unique solution $X \in D^{\text {ch }}$ given by

$$
X_{t}=\sum_{n \geq 0} I_{n}\left(f_{n}(\cdot, t)\right)
$$

where the kernels $f_{n}$ are given by

$$
f_{0}(t)=e^{b t}
$$

and for every $n \geq 1$,

$$
f_{n}\left(t_{1}, \ldots, t_{n}, t\right)=\frac{a^{n}}{n !} e^{b t} 1_{[0, t]}^{\otimes n}\left(t_{1}, \ldots, t_{n}\right) .
$$

Proof: The expression (17) of the kernels $f_{n}$ follows from Proposition 3.40 of [17]. One can also compute it easily by the recurrence relation

$$
f_{0}(t)=e^{b t}, f_{n}\left(t_{1}, \ldots, t_{n}, t\right)=a \tilde{f}_{n-1}\left(t_{1}, \ldots, t_{n-1}, t_{n}\right) 1_{[0, t]}\left(t_{n}\right), \quad \forall n \geq 1 .
$$

We only then need to prove that $f_{n} \in|\mathcal{H}(\alpha)|^{\otimes n+1}$ (if $\alpha>\frac{1}{2}$ ) and $f_{n} \in \mathcal{H}(\alpha)^{\otimes n+1}$ (if $\alpha<\frac{1}{2}$ ) and that the sum (10) converges.

If $\alpha>\frac{1}{2}$, this follows easily from the inclusion (7), since

$$
\left\|\tilde{f}_{n}\right\|_{|\mathcal{H}(\alpha)| \otimes n+1} \leq \operatorname{cst}\left\|\tilde{f}_{n}\right\|_{L^{2}\left([0, T]^{n+1}\right)}
$$

and we can reduce to the classical situation $\left(\alpha=\frac{1}{2}\right)$ where the result is known.

If $\alpha<\frac{1}{2}$, then we need a new proof because the norm $\mathcal{H}(\alpha)$ is bigger than the norm $L^{2}$. Let us show that the kernel $f_{n}$ given by (17) (viewed as a function of $n+1$ variables $\left.t_{1}, \ldots t_{n}, t\right)$ belongs to the space $\mathcal{H}(\alpha)^{\otimes n+1}$. Here we can adapt an argument used in [20]. We will show that

$$
K^{*, n+1} f_{n} \in L^{2}\left([0, T]^{n+1}\right)
$$

where $K^{*, n}$ is the $n$ times tensor product of $K^{*}$. It holds, by applying first the operator $K^{*}$ to the variables $t_{1}, \ldots, t_{n}$ and then to the variable $t$,

$$
K^{*, n+1} f_{n}=\frac{a^{n}}{n !} K^{*} e^{b t}\left(K^{*, n}\left(1_{[0, t]}^{\otimes n}\right)\right)
$$


and therefore, since

$$
\left\|K^{*, n}\left(1_{[0, t]}^{\otimes n}\right)\right\|_{L^{2}\left([0, T]^{n}\right)}^{2}=\left\|K^{*}\left(1_{[0, t]}\right)\right\|_{L^{2}\left([0, T]^{n}\right)}^{2 n}=t^{2 n \alpha}
$$

we get

$$
\begin{aligned}
\left\|K^{*, n+1} f_{n}\right\|_{L^{2}\left([0, T]^{n+1}\right)} & =\frac{|a|^{n}}{n !}\left\|K^{*}\left(e^{b t} t^{2 n \alpha}\right)\right\|_{L^{2}([0, T])} \\
& =\frac{|a|^{n}}{n !}\left\|\sum_{k \geq 0} \frac{|b|^{k}}{k !} K^{*}\left(t^{k+2 \alpha n}\right)\right\|_{L^{2}([0, T])}
\end{aligned}
$$

Since for every $k \geq 1$, the function $t^{k+2 \alpha n}$ is Lipschitz, then we have, using (9)

$$
\begin{aligned}
\left\|K^{*}\left(t^{k+2 \alpha n}\right)\right\|_{L^{2}([0, T])} \leq & C(\alpha, T)(k+2 \alpha n)\left[\int_{0}^{T} K^{\alpha}(T, t)^{2} t^{4 n \alpha+2 k} d t\right. \\
& \left.+\int_{0}^{T}\left(\int_{t}^{T}(r-t) \frac{\partial K^{\alpha}}{\partial r}(r, t) d r\right)^{2} d t\right]^{1 / 2} \\
\leq & C(\alpha, T)(k+2 \alpha n) T^{k+\alpha(2 n+1)} .
\end{aligned}
$$

This implies that

$$
\left\|K^{*, n+1} f_{n}\right\|_{L^{2}\left([0, T]^{n+1}\right)} \leq \operatorname{cst} \frac{|a|^{n}}{n !} T^{\alpha(2 n+1)} .
$$

The function $f_{n}$ being symmetric in the first $n$ variables, we have

$$
\tilde{f}_{n}\left(t_{1}, \ldots, t_{m+1}\right)=\frac{1}{m+1} \sum_{i=0}^{m+1} f_{n}\left(t_{1}, \ldots, t_{m+1}^{i}, \ldots, t_{m}\right)
$$

where $t_{m+1}^{i}$ means that $t_{m+1}$ is on the position $i$. Clearly the bound (19) holds for $\tilde{f}_{n}$. By the above estimate, it is not difficult to see that the sum (10) is convergent because

$$
\sum_{n \geq 0}(n+1) !\left\|\tilde{f}_{n}\right\|_{\mathcal{H}(\alpha) \otimes n+1}^{2} \leq \operatorname{cst} \sum_{n \geq 0} \frac{a^{2 n}}{n !} T^{2 \alpha(2 n+1)}<\infty .
$$

The uniqueness of the solution in $D^{c h}$ is obvious because, if there are two solutions, then the kernels of the chaotic expansion verifies both the relation (18).

In fact, we have

Corollary 1 The unique solution in $D^{\text {ch }}$ of the equation $(15)$ is given by

$$
X_{t}=\exp \left(a B_{t}^{\alpha}-\frac{a^{2}}{2} t^{2 \alpha}+b t\right) \text {. }
$$


Proof: Formula (20) was already proved in [9], page 117. But, to compare to the twoparameter case, let us nevertheless show how (20) is obtained in the particular case where $b=0$. Consider the equation

$$
X_{t}=1+\int_{0}^{t} a X_{s} \delta B_{s}^{\alpha}, \quad t \in[0, T]
$$

and let, for every $t \in[0, T]$

$$
X_{t}=\sum_{n \geq 0} I_{n}\left(f_{n}(\cdot, t)\right)
$$

be the chaotic expression of $X$. Equation (21) can be rewritten as

$$
\sum_{n \geq 0} I_{n}\left(f_{n}(\cdot, t)\right)=1+a \sum_{n \geq 0} I_{n+1}\left(f_{n}\left(\widetilde{*, \star)} 1_{[0, t]}(\star)\right)\right.
$$

where $\cdot$ represents $n$ variables, $\star$ denotes one variable and $f_{n}\left(\cdot \widetilde{, \star)} 1_{[0, t]}(\star)\right.$ denotes the symmetrization of the function $f_{n}(\cdot, \star) 1_{[0, t]}(\star)$ in $n+1$ variables.

By identifying the corresponding Wiener chaos, we easily get

$$
f_{0}(t)=1, \quad f_{1}\left(t_{1}, t\right)=a 1_{[0, t]}\left(t_{1}\right)
$$

and

$$
f_{2}\left(t_{1}, t_{2}, t\right)=\frac{a^{2}}{2}\left(1_{\left[0, t_{1}\right]}\left(t_{2}\right) 1_{[0, t]}\left(t_{1}\right)+1_{\left[0, t_{2}\right]}\left(t_{1}\right) 1_{[0, t]}\left(t_{2}\right)\right)=\frac{a^{2}}{2} 1_{[0, t]}^{\otimes 2}\left(t_{1}, t_{2}\right) .
$$

By induction we will get for every $n \geq 1$

$$
f_{n}\left(t_{1}, t_{2}, \ldots, t_{n}\right)=\frac{a^{n}}{n !} \sum_{i=0}^{n} 1_{\left[0, t_{i}\right]}^{\otimes n-1}\left(\hat{t}_{i}\right) 1_{[0, t]}\left(t_{i}\right)=\frac{a^{n}}{n !} 1_{[0, t]}^{\otimes n}\left(t_{1}, \ldots, t_{n}\right)
$$

where by $\hat{t}_{i}$ we denoted the vector $\left(t_{1}, \ldots, t_{n}\right)$ with $t_{i}$ missing. Therefore, we can express the solution of (21) as

$$
X_{t}=\sum_{n \geq 0} \frac{a^{n}}{n !} I_{n}\left(1_{[0, t]}^{\otimes n}\right)=\exp \left(a B_{t}^{H}-\frac{a^{2}}{2}\left\|1_{[0, t]}\right\|_{\mathcal{U}}^{2}\right)
$$

where for the last equality we refer e.g. to [5].

Remark 1 In Skorohod setting, it is difficult, in general, to write an Euler's type scheme associated to the equation $X_{t}=x_{0}+\int_{0}^{t} \sigma\left(X_{s}\right) \delta B_{s}^{\alpha}$, even if $\alpha \geq 1 / 2$. Indeed, by using the integration by parts for the Skorohod integral $\delta$ and the Malliavin derivative $D$ (see 14]) $\delta(F u)=F \delta(u)-\langle D F, u\rangle_{\mathcal{H}(\alpha)}$ and by assuming that we approximate $X_{(k+1) / n}$ by $X_{k / n}+\int_{k / n}^{(k+1) / n} \sigma\left(X_{k / n}\right) \delta B_{s}^{\alpha}$ (as in the case $\alpha=1 / 2$ ), one obtains

$$
\widehat{X}_{(k+1) / n}^{(n)}=\widehat{X}_{k / n}^{(n)}+\sigma\left(\widehat{X}_{k / n}^{(n)}\right)\left(B_{(k+1) / n}^{\alpha}-B_{k / n}^{\alpha}\right)-\sigma^{\prime}\left(\widehat{X}_{k / n}^{(n)}\right)\left\langle D \widehat{X}_{k / n}^{(n)}, 1_{[k / n,(k+1) / n]}\right\rangle_{\mathcal{H}(\alpha)} .
$$


The problem is that the quantity $D \widehat{X}_{k / n}^{(n)}$ appears and that it is difficult to compute it directly (without knowing the solution). Moreover, standard Euler scheme do not apply here because the $L^{2}$-norm of the Skorohod integral involves the first Malliavin derivative which involves the second Malliavin derivative etc. and we cannot have closable formulas. In the linear case, taking advantage from the fact that we know explicitly the solution, we can see what the correct Euler scheme should be. Indeed, since we have $D X_{k / n}=a X_{k / n} \mathbf{1}_{[0, k / n]}$ (see Corollary 1 above), a natural Euler's type scheme associated to (15) with $b=0$ is

$$
\widehat{X}_{(k+1) / n}^{(n)}=\widehat{X}_{k / n}^{(n)}+a \widehat{X}_{k / n}^{(n)}\left(B_{(k+1) / n}^{\alpha}-B_{k / n}^{\alpha}\right)-\frac{a^{2}}{2} \widehat{X}_{k / n}^{(n)}\left[\left(\frac{k+1}{n}\right)^{2 H}-\left(\frac{k}{n}\right)^{2 H}-\frac{1}{n^{2 H}}\right] .
$$

In fact, it is not very difficult to prove that $\left(\widehat{X}_{1}^{(n)}\right)$ converges in $\mathrm{L}^{2}(\Omega)$ if and only if $\alpha \geq 1 / 2$ and that, in the case where $\alpha>1 / 2$, the limit is $\exp \left(a B_{1}^{\alpha}-\frac{a^{2}}{2}\right)$. We refer to [1]] for other questions about schemes associated to stochastic equations driven by a fractional Brownian motion.

As we have seen, the solution of (21) is an exponential, hence positive, function. We will show that the situation is different in the two-parameter case.

Before that, let us consider the equation corresponding to (15) in the two-parameter case

$$
X_{z}=1+\int_{[0, z]} a X_{r} \delta W_{r}^{\alpha, \beta}+\int_{[0, z]} b X_{r} d r
$$

where $z=(s, t) \in[0, T]^{2}$ and $W^{\alpha, \beta}$ is a fractional Brownian sheet with Hurst parameters $\alpha, \beta \in(0,1)$.

We will denote now by $D^{c h, 2}$ the class of functionals that can be represented as a serie of multiple stochastic integrals with respect to $W^{\alpha, \beta}$ (that is, $D^{c h, 2}$ is the two-parameter equivalent of $D^{c h}$ ). In the next proposition, we show that (25) admits a unique solution in this space:

Proposition 2 Let us denote by $\mathcal{A}_{n}$ the set $\left\{\left(z_{1}, \ldots, z_{n}\right) \in\left(\mathbb{R}^{2}\right)^{n}: \exists \sigma \in \mathfrak{S}_{n}, z_{\sigma(1)} \leq \ldots \leq\right.$ $\left.z_{\sigma(n)}\right\}$ (in the one-parameter case: $\mathcal{A}_{n}=\mathbb{R}^{n}$ ). If $z \in \mathcal{A}_{n}$, we consider $\sigma=\sigma_{z} \in \mathfrak{S}_{n}$ such that $z_{\sigma(1)} \leq \ldots \leq z_{\sigma(n)}$. where

The equation (25) admits an unique solution $X \in D^{\text {ch, }, 2}$ given by $X_{z}=\sum_{n \geq 0} I_{n}\left(f_{n}(\cdot, z)\right)$

$$
\begin{aligned}
f_{n}\left(z_{1}, \ldots, z_{n}, z\right)= & \frac{a^{n}}{n !} h_{0}\left(b\left(s-s_{\sigma_{z}(n)}\right)\left(t-t_{\sigma_{z}(n)}\right)\right) \times \mathbf{1}_{\mathcal{A}_{n}}\left(z_{1}, \ldots, z_{n}\right) \mathbf{1}_{[0, z]}^{\otimes n}\left(z_{1}, \ldots, z_{n}\right) \\
& \times \prod_{1 \leq j \leq n} h_{0}\left(b\left(s_{\sigma_{z}(j)}-s_{\sigma_{z}(j-1)}\right)\left(t_{\sigma_{z}(j)}-t_{\sigma_{z}(j-1)}\right)\right)
\end{aligned}
$$

with $z=(s, t), z_{i}=\left(s_{i}, t_{i}\right)$ and $h_{0}(x)=\sum_{n=0}^{\infty} \frac{x^{n}}{(n !)^{2}}$. We also used the convention that $\sigma_{z}(0)=0$ and $z_{0}=(0,0)$. 
Proof: We only prove the algebraic part (26) of the Proposition. Indeed, the fact that the kernels $f_{n}$ belongs to $\mathcal{V}^{\otimes n+1}$ did not present new difficulties with respect to the proofs of Propositions 1 and 3. Thus, we return to these proofs for this point. Let us write

$$
X_{z}=\sum_{n \geq 0} I_{n}\left(f_{n}(\cdot, z)\right)
$$

Here, $I_{n}$ is the $n$-order Wiener-Itô multiple integral with respect to the fractional Brownian sheet $W^{\alpha, \beta}$ and $f_{n} \in L^{2}\left([0, T]^{2 n}\right)$. ¿From (25) we have that $f_{0}(z)=h_{0}(b s t)$ and for $n \geq 1$,

$$
f_{n}\left(z_{1}, \ldots, z_{n}, z\right)=a f_{n-1}\left(z_{1}, \widetilde{\left., z_{n}\right)} 1_{[0, z]}\left(z_{n}\right)+b \int_{[0, z]} f_{n}\left(z_{1}, \ldots, z_{n}, r\right) d r .\right.
$$

Let $n=1$. We therefore have

$$
f_{1}\left(z_{1}, z\right)=a h_{0}\left(b s_{1} t_{1}\right) \mathbf{1}_{[0, z]}\left(z_{1}\right)+b \int_{[0, z]} f_{1}\left(z_{1}, r\right) d r
$$

and

$$
f_{1}\left(z_{1}, z\right)=a h_{0}\left(b s_{1} t_{1}\right) h_{0}\left(b\left(s-s_{1}\right)\left(t-t_{1}\right)\right) \mathbf{1}_{[0, z]}\left(z_{1}\right)
$$

hence (26) is satisfied. If $n=2$ it holds that

$$
\begin{gathered}
f_{2}\left(z_{1}, \widetilde{z}_{2}, z_{3}\right) \mathbf{1}_{[0, z]}\left(z_{3}\right)=\frac{1}{2}\left(a h_{0}\left(b s_{1} t_{1}\right) h_{0}\left(b\left(s_{2}-s_{1}\right)\left(t_{2}-t_{1}\right)\right) \mathbf{1}_{0 \leq z_{1} \leq z_{2} \leq z}\right. \\
\left.+a h_{0}\left(b s_{2} t_{2}\right) h_{0}\left(b\left(s_{1}-s_{2}\right)\left(t_{1}-t_{2}\right)\right) \mathbf{1}_{0 \leq z_{2} \leq z_{1} \leq z}\right) .
\end{gathered}
$$

Since

$$
f_{2}\left(z_{1}, z_{2}, z\right)=a f_{1}\left(z_{1}, \widetilde{\left.z_{2}\right) \mathbf{1}_{[0, z]}}\left(z_{2}\right)+b \int_{[0, z]} f_{1}\left(z_{1}, r\right) d r\right.
$$

we deduce that

$$
\begin{gathered}
f_{2}\left(z_{1}, z_{2}, z\right)=\frac{a^{2}}{2}\left(h_{0}\left(b s_{1} t_{1}\right) h_{0}\left(b\left(s_{2}-s_{1}\right)\left(t_{2}-t_{1}\right)\right) h_{0}\left(b\left(s-s_{2}\right)\left(t-t_{2}\right)\right) \mathbf{1}_{0 \leq z_{1} \leq z_{2} \leq z}\right. \\
\left.+h_{0}\left(b s_{2} t_{2}\right) h_{0}\left(b\left(s_{1}-s_{2}\right)\left(t_{1}-t_{2}\right)\right) h_{0}\left(b\left(s-s_{1}\right)\left(t-t_{1}\right)\right) \mathbf{1}_{0 \leq z_{2} \leq z_{1} \leq z}\right)
\end{gathered}
$$

and again (26) is verified. The above computations can be easily extended to an induction argument.

Let us now discuss the case $b=0$ :

Proposition 3 The equation

$$
X_{z}=1+\int_{[0, z]} a X_{r} \delta W_{r}^{\alpha, \beta}, \quad z \in[0, T]^{2}
$$

admits an unique solution $X \in D^{\text {ch,2 }}$ given by $X_{z}=\sum_{n \geq 0} I_{n}\left(f_{n}(\cdot, z)\right)$ where

$$
f_{n}\left(\rho_{1}, \ldots, \rho_{n}, z\right)=\frac{a^{n}}{n !} \sum_{i=1}^{n} 1_{\left[0, \rho_{i}\right]}^{\otimes n-1}\left(\hat{\rho}_{i}\right) 1_{[0, z]}\left(\rho_{i}\right) .
$$


Proof: Let us write

$$
X_{z}=\sum_{n \geq 0} I_{n}\left(f_{n}(\cdot, z)\right)
$$

¿From the equivalent of relation (22) in the two-parameter case, we obtain

$$
f_{0}(z)=1, \quad f_{1}\left(\rho_{1}, z\right)=a 1_{[0, z]}\left(\rho_{1}\right)
$$

and in general relation (28) holds. Since $\mathcal{A}_{n} \neq\left(\mathbb{R}^{2}\right)^{n}$ (recall that $\mathcal{A}_{n}$ is defined in Proposition 2 ), note that this last expression is not equal to $\frac{a^{n}}{n !} 1_{[0, z]}^{\otimes n}\left(\rho_{1}, \ldots \rho_{n}\right)$ as in the one-parameter case (see Corollary 1).

Let us now prove that the kernel $f_{n}$ belongs to the space $\mathcal{V}^{\otimes n+1}$. When the Hurst parameters $\alpha$ and $\beta$ are bigger than $\frac{1}{2}$, then we can use (13) and then refer to the standard case of the Brownian sheet. We will thus only discuss the case $\alpha, \beta<\frac{1}{2}$; the case $\alpha>\frac{1}{2}$ and $\beta<\frac{1}{2}$ will be a mixture of the other two cases. We use the induction. We will illustrate first the case $n=2$. We check that $1_{[0, z]}\left(z_{2}\right) 1_{\left[0, z_{2}\right]}\left(z_{1}\right)$ belongs to $\mathcal{H}^{(2)^{\otimes 3}}$. This actually reduces to proving that

$$
1_{[0, t]}\left(t_{2}\right) 1_{\left[0, t_{2}\right]}\left(t_{1}\right) \in \mathcal{H}(\alpha)^{\otimes 3} .
$$

Let us apply the operator $K^{*, 3}$ in three steps: first to the variable $t_{1}$, then to the variable $t$ and then to $t_{2}$. It holds that

$$
\begin{aligned}
\left\|K^{*, 3}\left(1_{[0, t]}\left(t_{2}\right) 1_{\left[0, t_{2}\right]}\left(t_{1}\right)\right)\right\|_{L^{2}\left([0, T]^{3}\right)}^{2} & =\left\|K^{*, 2}\left(t_{2}^{2 \alpha} 1_{[0, t]}\left(t_{2}\right)\right)\right\|_{L^{2}\left([0, T]^{2}\right)}^{2} \\
& =\left\|K^{*, 1}\left(t_{2}^{2 \alpha}\left(T-t_{2}\right)^{2 \alpha}\right)\right\|_{L^{2}([0, T])}^{2}
\end{aligned}
$$

and to conclude we refer to Proposition 3.6 in [3]: it is a straightforward consequence of Lemma 4.3 in [3] that $\left(T-t_{2}\right)^{2 \alpha}\left(B^{\alpha}\right)^{2}$ belongs to the extended domain of the divergence and therefore its expectation is in $\mathcal{H}(\alpha)$.

We will show now that the kernel $1_{\left[0, \rho_{i}\right]}^{\otimes n-1}\left(\hat{\rho}_{i}\right) 1_{[0, z]}\left(\rho_{i}\right)$ has a finite norm in $\mathcal{H}^{(2)^{\otimes n+1}}$ by assuming that the result is true for $n$ variables. It suffices to check that the function of $n+1$ (real) variables

$$
1_{[0, t]}\left(t_{n}\right) 1_{\left[0, t_{n}\right]}\left(t_{n+1}\right) \ldots 1_{\left[0, t_{2}\right]}\left(t_{1}\right)
$$

belongs to $\mathcal{H}(\alpha)^{\otimes n+1}$ or, equivalently, the operator $K^{*, n+1}$ applied to the above function is in $L^{2}\left([0, T]^{n+1}\right)$. By applying first the operator $K^{*}$ to the variable $t_{1}$ it holds that

$$
\begin{aligned}
& \left\|K^{*, n+1}\left(1_{[0, t]}\left(t_{n}\right) 1_{\left[0, t_{n}\right]}\left(t_{n+1}\right) \ldots 1_{\left[0, t_{2}\right]}\left(t_{1}\right)\right)\right\|_{L^{2}\left([0, T]^{n+1}\right)}^{2} \\
= & \left\|K^{*, n}\left(1_{[0, t]}\left(t_{n}\right) 1_{\left[0, t_{n}\right]}\left(t_{n+1}\right) \ldots 1_{\left[0, t_{3}\right]}\left(t_{2}\right) t_{2}^{2 \alpha}\right)\right\|_{L^{2}\left([0, T]^{n}\right)}^{2} \\
= & \left\|K^{*}\left(t_{2}^{2 \alpha} g\left(t_{2}\right)\right)\right\|_{L^{2}([0, T])}^{2}
\end{aligned}
$$

where the function $t_{2} \rightarrow g\left(t_{2}\right):=\left\|K^{*, n-1}\left(1_{[0, t]}\left(t_{n}\right) 1_{\left[0, t_{n}\right]}\left(t_{n+1}\right) \ldots 1_{\left[0, t_{3}\right]}\left(t_{2}\right)\right)\right\|_{L^{2}\left([0, T]^{n-1}\right)}^{2}$ belongs to $\mathcal{H}(\alpha)$ by the induction hypothesis. Now, we refer to the proof of Proposition 3.6 in [3] for the fact that $g(\cdot) E\left(B .^{2}\right)$ has a finite norm in $\mathcal{H}(\alpha)$. 
It can actually be proved as above that

$$
\left\|1_{\left[0, \rho_{i}\right]}^{\otimes n-1}\left(\hat{\rho}_{i}\right) 1_{[0, z]}\left(\rho_{i}\right)\right\|_{\mathcal{H}^{(2)} \otimes n+1} \leq \frac{C^{n}}{n !}
$$

for every $n$ where $C$ is a positive constant. Now we can finish as in proof of Proposition 1.

We will need the following Girsanov theorem. Its proof will be given in the Appendix.

Lemma 1 For any $\varepsilon>0$, the process

$$
W_{s, t}^{\alpha, \beta, \varepsilon}=W_{s, t}^{\alpha, \beta}-\frac{s t}{\varepsilon}
$$

has the same law as a fractional Brownian sheet with parameters $\alpha, \beta$ under the new probability $P_{\varepsilon}$ given by

$$
\frac{d P^{\varepsilon}}{d P}=\exp \left(\frac{1}{\varepsilon} W_{T, T}^{\alpha, \beta}-\frac{1}{2 \varepsilon^{2}} \int_{[0, T]^{2}}\left(K_{\alpha, \beta}^{-1}(F(\cdot))(\rho)\right)^{2} d \rho\right)
$$

where $F(t, s)=t s$ and $K_{\alpha, \beta}$ is the operator associated to the kernel of the $W^{\alpha, \beta}$.

The solution of the equation (27) has a different behavior comparing to the oneparameter case (Corollary il). We prove actually below that the solution of (27) is almost surely negative on a non-negligible set. Note that the same problem has been studied in the case of the standard Brownian sheet in [15].

Proposition 4 Let $X$ be the unique solution to (27) in the space $D^{c h, 2}$. Then

$$
P\left\{\text { there exists an open set } \Delta \subset[0, T]^{2} \text { such that } X_{z}<0 \text { for all } z \in \Delta\right\}>0 \text {. }
$$

Remark 2 It seems that the following statement is also true: there exists an open set $\Delta \subset[0, T]^{2}$ such that

$$
P\left\{X_{z}<0 \text { for all } z \in \Delta\right\}>0 .
$$

A way to obtain it would be to prove that we have (keeping the same notations as in the proof of Proposition (1)

$$
E\left[\sup _{s, t \in[0, T]}\left|Y_{s, t}^{\varepsilon}-h_{0}(-a s t)\right|^{2}\right] \rightarrow_{\varepsilon \rightarrow 0} 0
$$

instead of (35). Although it seemed possible to us to show this more restrictive convergence, we prefered, for the simplicity, only to prove (31). 
Proof of Proposition 车: Note that the deterministic equation

$$
g(s, t)=1+\int_{0}^{s} \int_{0}^{t} a g(u, v) d u d v
$$

admits the unique solution $g(s, t)=h_{0}($ ast $)$ with $h_{0}(x)=\sum_{n \geq 0} \frac{x^{n}}{(n !)^{2}}$ and that the function $h_{0}$ satisfy the property: there exists an open set $I=(-\beta,-\alpha)$ such that $h_{0}(x)<-\delta<0$ for any $x \in I$ (see [15], page 231).

Suppose $a>0$, fix $N>0$ and define the open set

$$
\tilde{\Delta}=\{(s, t), \alpha<a s t<\beta, 0<s, t<N\} .
$$

For every $\varepsilon>0$, consider

$$
X_{z}^{\varepsilon}=1+\int_{[0, z]} a \varepsilon X_{r}^{\varepsilon} \delta W_{r}^{\alpha, \beta}
$$

Thanks to Corollary 1, we know that the solution $X^{\varepsilon}$ of (3) is given by

$$
X_{z}^{\varepsilon}=\sum_{n \geq 0} \varepsilon^{n} I_{n}\left(f_{n}(\cdot, z)\right)
$$

where the kernels $f_{n}$ are given by $f_{n}\left(\rho_{1}, \ldots, \rho_{n}, z\right)=\frac{a^{n}}{n !} \sum_{i=1}^{n} 1_{\left[0, \rho_{i}\right]}^{\otimes n-1}\left(\hat{\rho}_{i}\right) 1_{[0, z]}\left(\rho_{i}\right)$. Let us consider the equation

$$
Y_{z}^{\varepsilon}=1+\int_{[0, z]} a \varepsilon Y_{r}^{\varepsilon} d W_{r}^{\alpha, \beta, \varepsilon}=1+\int_{[0, z]} a \varepsilon Y_{r}^{\varepsilon} d W_{r}^{\alpha, \beta}-\int_{[0, z]} a Y_{r}^{\varepsilon} d r
$$

and recall that, by Lemma 1, $W^{\alpha, \beta, \varepsilon}$ is a fractional Brownian sheet under $P_{\varepsilon}$. Now, we observe that

$$
K=\sup _{\varepsilon>0} \sup _{z} E\left|Y_{z}^{\varepsilon}\right|^{2}<\infty .
$$

In fact, to show that (34) holds is not difficult because it follows from Proposition 2 that the kernel of order $n$ appearing in the chaotic expression of the solution of (33) are of the form $\varepsilon^{n}$ multiplied to the kernel of order $n$ of the solution of (33) with $\varepsilon=1$. Then, $K \leq \sup _{z} E\left|Y_{z}^{1}\right|^{2}<\infty$.

Now, by (32) and (33) we have, for $z=(t, s)$,

$$
Y_{s, t}^{\varepsilon}-h_{0}(-a s t)=-a \int_{0}^{s} \int_{0}^{t}\left(Y_{u, v}^{\varepsilon}-h_{0}(-a u v)\right) d v d u+a \varepsilon \int_{0}^{s} \int_{0}^{t} Y_{u, v}^{\varepsilon} d W_{u, v}^{\alpha, \beta}
$$

and using the bound (34) and the Gronwall Lemma in the plane we obtain

$$
\sup _{s, t \in[0, T]} E\left[\left|Y_{s, t}^{\varepsilon}-h_{0}(-a s t)\right|^{2}\right] \rightarrow_{\varepsilon \rightarrow 0} 0 .
$$


Since $h_{0}(-a s t)<-\delta$ for $(s, t) \in \tilde{\Delta}$, it follows that

$$
P\left(Y_{z}^{\varepsilon}<0\right) \rightarrow_{\varepsilon \rightarrow 0} 1 \text {, uniformly on } z \in \tilde{\Delta} \text {. }
$$

Thus, for every $\varepsilon>0$ small enough

$$
P\left(Y_{z}^{\varepsilon}<0\right)>0, \quad \forall z \in \tilde{\Delta}
$$

and

$$
P_{\varepsilon}\left(Y_{z}^{\varepsilon}<0\right)=P\left(X_{z}^{\varepsilon}<0\right)>0, \quad \forall z \in \tilde{\Delta} .
$$

Since $W_{c_{1} s, c_{2} t}^{\alpha, \beta}$ has the same law as $c_{1}^{\alpha} c_{2}^{\beta} W_{s, t}^{\alpha, \beta}$ as process, we get that $X_{s, t}^{\varepsilon}$ is equal in law to $X_{\varepsilon^{2 \alpha} s, \varepsilon^{2 \beta} t}$. So, for $\varepsilon>0$ small enough,

$$
P\left(X_{\varepsilon^{2 \alpha} s, \varepsilon^{2 \beta} t}<0\right)>0, \quad \forall z=(s, t) \in \tilde{\Delta}
$$

and the conclusion follows.

\section{Appendix}

Proof of Lemma 1: The conclusion will follow from the Girsanov theorem for the fractional Brownian sheet (see Theorem 3 in [7]) if we show that the functions $F(s, t)=s t$ belongs to the space $I^{\alpha+\frac{1}{2}, \beta+\frac{1}{2}}\left(L^{2}\left([0, T]^{2}\right)\right)$ or equivalently,

$$
K_{\alpha, \beta}^{-1}(F(\cdot)) \in L^{2}\left([0, T]^{2}\right) .
$$

To show this, we will need the expression of its inverse operator in terms of fractional integrals and derivatives (see e.g. [7])

$$
K_{\alpha, \beta}^{-1} h(t, s)=t^{\alpha-\frac{1}{2}} s^{\beta-\frac{1}{2}} I^{\frac{1}{2}-\alpha, \frac{1}{2}-\beta}\left(t^{\frac{1}{2}-\alpha} s^{\frac{1}{2}-\beta} \frac{\partial^{2} h}{\partial s \partial t}\right), \quad \alpha, \beta<\frac{1}{2}
$$

and

$$
K_{\alpha, \beta}^{-1} h(t, s)=t^{\alpha-\frac{1}{2}} s^{\beta-\frac{1}{2}} D^{\alpha-\frac{1}{2}, \beta-\frac{1}{2}}\left(t^{\frac{1}{2}-\alpha} s^{\frac{1}{2}-\beta} \frac{\partial^{2} h}{\partial s \partial t}\right), \quad \alpha, \beta>\frac{1}{2}
$$

Here,

$$
I^{\alpha, \beta} f(x, y)=\frac{1}{\Gamma(\alpha) \Gamma(\beta)} \int_{0}^{x} \int_{0}^{y}(x-u)^{\alpha-1}(y-v)^{\beta-1} f(u, v) d u d v
$$

and

$$
D^{\alpha, \beta} f(x, y)=\frac{1}{\Gamma(1-\alpha) \Gamma(1-\beta)} \frac{\partial^{2}}{\partial x \partial y} \int_{0}^{x} \int_{0}^{y} \frac{f(u, v)}{(x-u)^{\alpha}(y-v)^{\beta}} d u d v
$$

with $\Gamma$ the Euler function.

For $\alpha, \beta \in\left(0, \frac{1}{2}\right)$ we have 


$$
\begin{aligned}
K_{\alpha, \beta}^{-1} F(t, s) & =t^{\alpha-\frac{1}{2}} s^{\beta-\frac{1}{2}} I^{\alpha-\frac{1}{2}, \beta-\frac{1}{2}}\left(t^{\frac{1}{2}-\alpha} s^{\frac{1}{2}-\beta}\right) \\
& =\frac{1}{\Gamma\left(\frac{1}{2}-\alpha\right) \Gamma\left(\frac{1}{2}-\beta\right)} t^{\alpha-\frac{1}{2}} s^{\beta-\frac{1}{2}} \int_{0}^{t} \int_{0}^{s}(t-u)^{-\frac{1}{2}-\alpha} u^{\frac{1}{2}-\alpha}(s-v)^{-\frac{1}{2}-\beta} v^{\frac{1}{2}-\beta} d v d u
\end{aligned}
$$

and this belongs to $L^{2}\left([0, T]^{2}\right)$.

If $\alpha, \beta \in\left(\frac{1}{2}, 1\right)$ then by (37) we can write

$$
\begin{aligned}
& K_{\alpha, \beta}^{-1} F(t, s) \\
= & t^{\alpha-\frac{1}{2}} s^{\beta-\frac{1}{2}} D^{\alpha-\frac{1}{2}, \beta-\frac{1}{2}}\left(t^{\frac{1}{2}-\alpha} s^{\frac{1}{2}-\beta}\right)(t, s) \\
= & t^{\alpha-\frac{1}{2}} s^{\beta-\frac{1}{2}} \frac{1}{\Gamma\left(\frac{3}{2}-\alpha\right) \Gamma\left(\frac{3}{2}-\beta\right)}\left[\frac{t^{\frac{1}{2}-\alpha} s^{\frac{1}{2}-\beta}}{t^{\alpha-\frac{1}{2}} s^{\beta-\frac{1}{2}}}\right. \\
& +\frac{\alpha-\frac{1}{2}}{s^{\beta-\frac{1}{2}}} \int_{0}^{t} \frac{t^{\frac{1}{2}-\alpha} s^{\frac{1}{2}-\beta}-u^{\frac{1}{2}-\alpha} s^{\frac{1}{2}-\beta}}{(t-u)^{\alpha+\frac{1}{2}}} d u \\
& +\frac{\beta-\frac{1}{2}}{t^{\alpha-\frac{1}{2}}} \int_{0}^{s} \frac{t^{\frac{1}{2}-\alpha} s^{\frac{1}{2}-\beta}-t^{\frac{1}{2}-\alpha} v^{\frac{1}{2}-\beta}}{(s-v)^{\beta+\frac{1}{2}}} d v \\
& \left.+\left(\alpha-\frac{1}{2}\right)\left(\beta-\frac{1}{2}\right) \int_{0}^{t} \int_{0}^{s} \frac{t^{\frac{1}{2}-\alpha} s^{\frac{1}{2}-\beta}-u^{\frac{1}{2}-\alpha} s^{\frac{1}{2}-\beta}-t^{\frac{1}{2}-\alpha} v^{\frac{1}{2}-\beta}+u^{\frac{1}{2}-\alpha} v^{\frac{1}{2}-\beta}}{(t-u)^{\alpha+\frac{1}{2}}(s-v)^{\beta+\frac{1}{2}}} d v d u\right] .
\end{aligned}
$$

Since

$$
\int_{0}^{t} \frac{t^{\frac{1}{2}-\alpha}-u^{\frac{1}{2}-\alpha}}{(t-u)^{\alpha+\frac{1}{2}}} d u=c(\alpha) t^{1-2 \alpha},
$$

it is not difficult to see that the above function is in $L^{2}\left([0, T]^{2}\right)$.

If $\alpha \in\left(0, \frac{1}{2}\right)$ and $\beta \in\left(\frac{1}{2}, 1\right)$, then we have

$$
\begin{aligned}
K_{\alpha, \beta}^{-1} F(t, s)= & C(\alpha, \beta) t^{\alpha-\frac{1}{2}} \int_{0}^{t}(t-u)^{-\frac{1}{2}-\alpha} u^{\frac{1}{2}-\alpha} d u \\
& \times s^{\frac{1}{2}-\beta}+\left(\beta-\frac{1}{2}\right) \int_{0}^{s} \frac{t^{\frac{1}{2}-\alpha} s^{\frac{1}{2}-\beta}-t^{\frac{1}{2}-\alpha} v^{\frac{1}{2}-\beta}}{(s-v)^{\beta+\frac{1}{2}}} d v
\end{aligned}
$$

and the conclusion is clearly a consequence of the above two cases. The proof of Lemma is done.

Acknowledgement. We want to thank the anonymous referee for a very careful and thorough reading of our note and for many helpful and constructive remarks. 


\section{References}

[1] E. Alòs, J.A. Léon and D. Nualart (2001): Stratonovich calculus for fractional Brownian motion with Hurst parameter less than $\frac{1}{2}$. Taiwanese Journal of Math. 4, 609-632.

[2] R. Buckdahn (1991): Linear stochastic Skorohod differential equations. Probab. Theory Rel. Fields 90, 223-240.

[3] P. Cheridito and D. Nualart (2005): Stochastic integration of divergence type with respect to the fractional Brownian motion with Hurst parameter $H \in\left(0, \frac{1}{2}\right)$. Ann. Inst. H. Poincar Probab. Statist. 41 (6), 1049-1081.

[4] L. Coutin and Z. Qian (2002): Stochastic analysis, rough path analysis and fractional Brownian motion. Prob. Theory Rel. Fields 122 (1), 108-140.

[5] T. E. Duncan, Y. Hu and B. Pasik-Duncan (2000): Stochastic calculus for fractional Brownian motion I. Theory. Siam J. Control Optim. 38 (2), 582-612.

[6] M. Errami, F. Russo (2003). n-covariation and symmetric SDEs driven by finite cubic variation process. Stoch. Processes and their Applications 104, 259-299.

[7] M. Erraoui, D. Nualart and Y. Ouknine (2003): Hyperbolic stochastic partial differential equations with additive fractional Brownian sheet. Stochastic and Dynamics 3, 121-139.

[8] D. Feyel, A. De La Pradelle (2003): Curvilinear integrals along enriched paths. Preprint Évry.

[9] Y.Z. Hu (2005): Integral transformations and anticipating calculus for fractional Brownian motions. Memoirs AMS 175.

[10] J.A. Leon and D. Nualart (2005): An extension of the divergence operator for Gaussian processes. Stoch. Processes and their Applications 115, 481-492.

[11] A. Neuenkirch and I. Nourdin (2006): Exact rate of convergence of some approximation schemes associated to SDEs driven by a fBm. Preprint Paris 6.

[12] I. Nourdin (2005): A simple theory for the study of SDEs driven by a fractional Brownian motion, in dimension one. Preprint Paris 6.

[13] I. Nourdin and T. Simon (2006): Correcting Newton-Côtes integrals by Lévy areas. Preprint Évry.

[14] D. Nualart (1995): The Malliavin calculus and related topics. Springer.

[15] D. Nualart (1987): Some remarks on a linear stochastic differential equation. Stat. and Probab. Letters 5, 231-234. 
[16] D. Nualart and A. Răsçanu (2002). Differential equations driven by fractional Brownian motion. Collect. Math. 53 (1), 55-81.

[17] V. Perez Abreu and C. Tudor (2002): A transfer principle for multiple stochastic fractional integrals. Bol. Soc. Mat. Mexicana 8 (3), 187-203.

[18] V. Pipiras and M. Taqqu (2000): Integration questions related to fractional Brownian motion. Probab. Theory Rel. Fields 118, 251-291.

[19] T. Sottinen and C.A. Tudor (2005): Parameter estimation for stochastic equations with fractional Brownian sheet. Preprint Helsinki.

[20] C.A. Tudor and F. Viens (2004): Itô formula for the fractional Brownian sheet using the extended divergence operator. Preprint Paris 1.

[21] M. Zähle (1998): Integration with respect to fractal functions and stochastic calculus. Prob. Theory Rel. Fields 111, 333-374. 